\title{
Leukopenia in Kidney Transplant Patients With the Association of Valganciclovir and Mycophenolate Mofetil
}

\author{
S. Brum, F. Nolasco, J. Sousa, A. Ferreira, M. Possante, J.R. Pinto, E. Barroso, and J.R. Santos
}

\begin{abstract}
Cytomegalovirus (CMV) is the most common viral infection after transplantation. Valganciclovir (VGC) is established for prophylaxis and treatment of CMV infections, but leukopenia which appears in $10 \%$ to $13 \%$ (severe in $4.9 \%$ ) is the principal side effect. We have recently noted an increased incidence of leukopenia and severe neutropenia among our renal transplant patients and thought to identify the associated factors. We conducted a retrospective analysis of all kidney transplantations performed between January 2005 and December 2006. All patients received mycophenolate mofetil (MMF), tacrolimus, and steroids. VGC was used for targeted prophylaxis and preemptive therapy of CMV infection, with doses adjusted to renal function. Of the 64 patients undergoing renal transplantation $13(20.3 \%)$ developed leukopenia within $3 \pm 2$ months after transplantation with severe neutropenia in 5 (7.8\%). All patients were on MMF and VGC (VGC $605 \pm 296 \mathrm{mg} / \mathrm{d}$ ). Leukopenia was significantly associated with simultaneous liver-kidney transplantation and with second kidney transplantations $(P<.01)$. The incidence of leukopenia was higher among patients under VGC since day 1 of transplantation $(P=.008)$ with maximal incidence observed among patients prescribed $900 \mathrm{mg} / \mathrm{d}$ as opposed to those on lower doses $(P<.01)$. There was no increase in CMV infection among patients with a low dose of VGC. No patient developed clinical CMV disease. In conclusion, VGC prophylaxis was associated with an increased frequency of leukopenia on MMF-tacrolimus treated patients or regimens. Low-dose VGC for CMV prophylaxis appeared to be as effective as high-dose treatment, and associated less frequently with leukopenia and neutropenia.
\end{abstract}

$\mathrm{C}$ YTOMEGALOVIRUS (CMV) is a major cause of morbidity and mortality among solid organ transplant recipients. ${ }^{1}$ Among recipients of kidney transplants, CMV infection and disease are associated with decreased allograft and patient survivals. ${ }^{2}$ Risk factors for CMV infection and disease include donor (D) and recipient (R) serostatus, with donor-seropositive, recipient-seronegative $(\mathrm{D}+/ \mathrm{R}-)$ as well as administration of antilymphocyte antibodies. ${ }^{3}$

Valganciclovir (VGC) is an L-valyl ester of ganciclovir with an oral bioavailability 10 times higher than oral ganciclovir, with the convenience of a once-daily oral dosing regimen. ${ }^{4}$ It was recently reported that among $\mathrm{D}+/ \mathrm{R}-$ patients, $900 \mathrm{mg} / \mathrm{d}$ of VGC was as clinically effective as $3 \mathrm{~g} / \mathrm{d}$ of oral ganciclovir to prevent CMV. ${ }^{5}$ Several studies have reported that low-dose $(450 \mathrm{mg} / \mathrm{d}) \mathrm{VGC}$ is as effective as oral ganciclovir for CMV prophylaxis. ${ }^{6-9}$ Present guidelines recommend $900 \mathrm{mg} / \mathrm{d}$ for CMV prophylaxis. ${ }^{10}$

Leukopenia has been the main side effect of VGC. A recent study showed that full VGC doses are associated with an important risk for neutropenia (12.5\%), in conjunction with a cyclosporine (CsA)/tacrolimus-mycophenolate mofetil (MMF)-based regimen. ${ }^{11}$ In our transplant unit, in the last years we have noticed a higher incidence of leukopenia among our patients, particularly upon the introduction of an immunosuppressive regimen based on MMF and tacrolimus associated with VGC. Some of the episodes have been associated with severe neutropenia requiring granulocyte-colony stimulating factor (G-CSF) treatment. The risks factors associated with VGC-induced leukopenia in this population are not clearly understood.

From the Departments of Transplantation (S.B., F.N., J.S., A.F., M.P., J.R.P., E.B.) and Nephrology (S.B., J.R.S.), Hospital Curry Cabral, Lisbon, Portugal.

Address reprint requests to Sandra Brum, Serviço de Nefrologia, Hospital Curry Cabral, Rua da Beneficência, 8, 1069 Lisboa, Portugal. Email: sfcbrum@gmail.com

(C) 2008 by Elsevier Inc. All rights reserved. 360 Park Avenue South, New York, NY 10010-1710 
Table 1. Drug Doses and Type of Transplant in Patients With leukopenia

\begin{tabular}{|c|c|c|c|c|}
\hline & \multicolumn{3}{|c|}{ Leukopenia } & Neutropenia \\
\hline & \multicolumn{3}{|c|}{$(13 / 64 ; 20.3 \%)$} & $(5 / 64 ; 7.8 \%)$ \\
\hline \multicolumn{5}{|l|}{ Transplant type } \\
\hline Deceased allograft ${ }^{\star}$ & \multicolumn{3}{|c|}{$7 / 55(12.7 \%)$} & 4/55 (7.3\%) \\
\hline Living donor graft $^{\star}$ & \multicolumn{3}{|c|}{ 1/3 (33.3\%) } & $1 / 3(33.3 \%)$ \\
\hline Liver-kidney grafts* & \multicolumn{3}{|c|}{$2 / 3(66.7 \%)$} & - \\
\hline \multirow[t]{2}{*}{ Second graft* } & \multicolumn{3}{|c|}{$3 / 3(100 \%)$} & - \\
\hline & $\begin{array}{l}\text { Leukopenia } \\
\qquad(\mathrm{n}=13)\end{array}$ & $\begin{array}{l}\text { Absence of Leukopenia } \\
\qquad(\mathrm{n}=51)\end{array}$ & $\begin{array}{l}\text { Leukopenia With Neutropenia } \\
\qquad(\mathrm{n}=5)\end{array}$ & $\begin{array}{l}\text { Leukopenia Without Neutropenia } \\
\qquad(\mathrm{n}=8)\end{array}$ \\
\hline \multicolumn{5}{|l|}{ Drug dosage } \\
\hline Initial dose MMF (mg/d) & $1500 \pm 729^{\dagger}$ & $1824 \pm 442^{\dagger}$ & $2000 \pm 0^{\dagger}$ & $1188 \pm 788^{\dagger}$ \\
\hline Dose MMF at incidence $(\mathrm{mg} / \mathrm{d})$ & $673 \pm 373$ & & $719 \pm 432^{\ddagger}$ & $600 \pm 285^{\ddagger}$ \\
\hline Dose VGC at incidence (mg/d) & $605 \pm 296$ & & $675 \pm 318^{\ddagger}$ & $563 \pm 294^{\ddagger}$ \\
\hline
\end{tabular}

${ }^{\star} P=.0179$.

${ }^{\dagger} P<.05$.

${ }^{\ddagger} P>.05$.

\section{MATERIALS AND METHODS}

We retrospectively analyzed 64 kidney transplantations performed from January 2005 to December 2006, included 28 females (43.8\%) and 36 males $(56.3 \%)$ of overall mean age of $48.06 \pm 12.8$ years, including 3 who received simultaneous kidney-liver allografts, 3 living kidney donor allografts, 3 second kidney allografts, and 55 deceased donor kidney allografts.

All 64 patients received corticosteroids, MMF, and tacrolimus. Corticosteroids were given preoperatively at a dose of $500 \mathrm{mg}$ of methylprednisolone, as well as on days 1 and 2 posttransplantation, followed by oral prednisolone. MMF was started at $1000 \mathrm{mg}$ twice daily and reduced to 500 to $750 \mathrm{mg}$ twice daily by 1 month. Tacrolimus was initially prescribed at $0.1 \mathrm{mg} / \mathrm{kg} / \mathrm{d}$ divided into 2 doses, with adjustments based on trough blood levels. Induction regimens included basiliximab $20 \mathrm{mg}$ on day 0 and day 4 posttransplantation or T-lymphocyte immune globulin (ATG; $2-4 \mathrm{mg} / \mathrm{kg} / \mathrm{d}$ ) with reduced doses of tacrolimus and MMF for the first week. Six patients received induction therapy with ATG and 56 with basiliximab.

VGC was prescribed to high-risk patients, as targeted prophylaxis, for $\mathrm{D}+/ \mathrm{R}$ - or under ATG, and as preemptive therapy. VGC was adjusted for renal function, and reduced if leukopenia or thrombocytopenia was present.

For the purpose of this study, leukopenia was defined as a total white blood cell count of $<3000 / \mathrm{mm}^{3}$, and neutropenia as a neutrophil count $\leq 500 / \mathrm{mm}^{3}$ requiring treatment with GM-CSF (300 $\mu \mathrm{g} / \mathrm{d}$ until recovery). CMV infection was defined as a positive antigenemia laboratory test.

Data were analyzed using SPSS. Chi-square or Fisher exact test and Student $t$ test were performed with statistical significance set as $P<.05$.

\section{RESULTS}

Leukopenia was observed in 13 patients (20.3\%; Table 1) at a mean of $3 \pm 2$ months after transplantation. It occurred in $7 / 55$ recipients $(12.7 \%)$ of deceased donor kidney allografts; $1 / 3(33.3 \%)$ of living kidney donor allografts; $2 / 3(66.7 \%)$ of simultaneous liver-kidney grafts; and 3/3 (100\%) of second transplant allografts. Patients receiving second allografts and simultaneous liver and kidney grafts displayed a higher frequency $\left(\chi^{2}, P<.01\right)$. Severe neutropenia was detected in $7.8 \%$ of patients, requiring G-CSF (Table 1). No infections were detected during the study period. We found no increased incidence of leukopenia among patients on ATG.

All 13 patients were on VGC $(605 \pm 296 \mathrm{mg} / \mathrm{d}$; Table 1$)$; $5 / 13$ patients with severe neutropenia had received higher doses of VGC $(675 \pm 318 \mathrm{vs} 563 \pm 294 \mathrm{mg} / \mathrm{d})$, although the difference was not significant. The dose of MMF did not show significance, although higher doses of MMF were found among patients with neutropenia (719 \pm 432 vs $600 \pm 285 \mathrm{mg} / \mathrm{d} ; P>.05)$.

Patients with leukopenia underwent lower in of MMF doses in the first days after transplantation: $1500 \pm 729$ vs $1824 \pm 442 \mathrm{mg} / \mathrm{d}(P=.046)$.

VGC was started in 15 patients on day 1 of transplantation: 4 with a high dose $(900 \mathrm{mg} / \mathrm{d})$ and the others a lower dose of $450 \mathrm{mg} / \mathrm{d}$ due to renal insufficiency and/or concomitant administration of ATG. Seven patients developed leukopenia $(7 / 15 ; 46.7 \%)$. In contrast, leukopenia was found in only 6 of 49 patients who did not receive VGC from day 1 (6/49; $12.2 \%$; Fisher, $P=.008)$.

The maximal incidence of leukopenia was observed in patients prescribed $900 \mathrm{mg} / \mathrm{d}(3 / 4 ; 75 \%)$. In contrast a lower incidence was observed among patients on $450 \mathrm{mg} / \mathrm{d}(4 / 11$; $36.7 \%)$ or who had not started prophylaxis on day $1(6 / 49$; $\left.12.2 \% ; \chi^{2}, P=.01\right)$.

We failed to observe an association of leukopenia with the tacrolimus level or renal function at the time of leukopenia, at 2 months posttransplantation, or at the last observation (data not shown).

All patients with leukopenia had no positive antigenemia laboratory test. In 4 patients, a positive antigenemia laboratory test was detected $1.4 \pm 0.5$ months before leukopenia. These patients were started on $900 \mathrm{mg} / \mathrm{d}$.

Leukopenia was not correlated with CMV serostatus of the donor or the recipient, nor with a previous positive antigenemia laboratory test. 


\section{DISCUSSION}

Leukopenia was observed among $20.3 \%$ of out patients with severe neutropenia in $7.8 \%$. This is a higher incidence than that previously noted with ganciclovir. Despite these facts, no infectious complications were observed. Once leuckpenia was detected, VGC was immediately tapered or eventually stopped, and the dose of MMF also reduced. Among patients with neutropenia, MMF and VGC were stopped, and G-CSF prescribed. Patients rapidly recovered leukocyte counts within 2 to 3 days with this strategy. It is noteworthy that VGC was reintroduced in 2 patients with relapse of leukopenia within 2 weeks.

Other studies have reported incidences of leukopenia ranging from $10 \%$ to $55.5 \%$. Neutropenia has been detected in $4.9 \%$ to $37.5 \% .^{12,13}$ It is noteworthy that higher incidences of leukopenia and/or neutropenia have been found in studies involving a small number of patients, probably causing some bias. ${ }^{12,13}$ In contrast to our study, Rerolle et $\mathrm{al}^{12}$ observed severe infectious complications associated with neutropenia. The difference from our patients may result from the intensive monitoring, and prompt use of G-CSF.

The risks factors associated with VGC-induced leukopenia are not clear. To our knowledge, there is only one study that looked at risk factors associated with VGC-induced leukopenia. ${ }^{13}$ Chen et al ${ }^{13}$ demonstrated that VGC toxicity was related to low body mass index, suggesting that dose should be adjusted to body weight, as racial or genetic factors may be important.

Our data showed that leukopenia was associated with the following factors: (1) high dose of VGC; (2) type of allograft; (3) starting prophylaxis on day 1, and in the case of neutropenia, possibly higher doses of MMF.

Some authors have suggested that low doses of VGC are as appropriate as full doses of $\mathrm{VGC}^{14}$; others have shown that VGC $450 \mathrm{mg} / \mathrm{d}$ provides equivalent drug exposure as oral ganciclovir $1000 \mathrm{mg}$ three times a day. ${ }^{15}$

Our results showed that VGC $450 \mathrm{mg} / \mathrm{d}$ was safe and effective for CMV prophylaxis. However, further studies comparing VGC $900 \mathrm{vs} 450 \mathrm{mg} / \mathrm{d}$ are needed to determine the optimal CMV prophylaxis regimen.

In conclusion, patients under VGC treatment must have intensive surveillance, because leukopenia and neutropenia show increased incidences with associated risks, requiring changes in immunosuppression. The risk of leukopenia is reduced with lower VGC dosages without an apparent change in efficacy.

\section{REFERENCES}

1. Fishman JA, Rubin RH: Infection in organ-transplant recipients. N Engl J Med 338:1741, 1998

2. Sagedel S, Hartmann A, Nordal KP, et al: Impact of early cytomegalovirus infection and disease on long-term recipient and kidney graft survival. Kidney Int 66:329, 2004

3. Guidelines of the American Society of Transplantation. Cytomegalovirus. Am J Transplant 4(suppl 10):51, 2004

4. Pescovitz MD, Rabkin, Merion RM, et al. Valgancyclovir results in improved oral absorption of ganciclovir in liver transplant recipients. Antimicrob Agents Chemother 44:2811, 2000

5. Paya C, Humar A, Dominguez E, et al: Efficacy and safety of valgancyclovir vs. oral ganciclovir for prevention of cytomegalovirus disease in solid organ transplant recipients. Am J Transplant 4:611, 2004

6. Akalin E, Sehgal V, Ames S, et al: Cytomegalovirus disease in high-risk transplant recipients despite ganciclovir or valganciclovir prophylaxis. Am J Transplant 3:731, 2003

7. Gabardi S, Magee CC, Baroletti SA, et al: Efficacy and safety of low dose valgancyclovir for prevention of cytomegalovirus disease in renal transplants recipients: a single-center, retrospective analysis. Pharmacotherapy 24:1323, 2004

8. Keven K, Basu A, Tan HP, et al: Cytomegalovirus prophylaxis using oral ganciclovir or valgancyclovir in kidney and pancreaskidney transplantation under antibody preconditioning. Transplant Proc 36:3107, 2004

9. Weng FL, Patel A, Wanchoo R, et al: Oral ganciclovir versus low-dose valgancyclovir for prevention of cytomegalovirus disease in recipients of kidney and pancreas transplants. Transplantation 83:290, 2007

10. Preiksaitis JK, Brennan DC, Fishman J, et al: Canadian Society of Transplantation consensus workshop on cytomegalovirus management in solid organ transplantation final report. Am J Transplant 5:218, 2005

11. Pedroche CD, Lumbreras C, Juan R, et al: Valganciclovir preemptive therapy for the prevention of cytomegalovirus disease in high-risk seropositive solid-organ transplant recipient. Transplantation 82:30, 2006

12. Rerolle J, Szelag J, Meur Y: Unexpected rate of severe leucopenia with the association of mycophenolate mofetil and valganciclovir in kidney transplant recipients. Nephrol Dial Transplant 22:671, 2007

13. Chen I, Chang H, Hsu C, et al: Correlation between body mass index and leucopenia after administration of valganciclovir for cytomegalovirus infection in Chinese cardiac recipients. Circ J 71:968, 2007

14. Pescovitz M: Is low dose valganciclovir the same as appropriate-dose valganciclovir. Transplantation 84:126, 2007

15. Chamberlain C, Mannon R, Penzac S, et al: Pharmacokinetics of low and maintenance dose valganciclovir in kidney transplant recipients. Am J Transplant 6 (suppl 2):266, 2006 